\title{
$X-R a y$ Powder Diffraction Study of the R-Phase in a Ti50Ni48Fe2 Alloy by a New Calibration Method
}

\author{
G. Airoldi, G. Carcano*, G. Riva** and M. Vanelli \\ Dipartimento di Fisica, Universita' di Milano, via Celoria 16, 20133 Milano, Italy \\ * ITM-CNR, Area della Ricerca di Milano, via Bassini 15, 20133 Milano, Italy \\ ** INFM, Unita di Milano Universita', via Celoria 16, 20133 Milano, Italy
}

\begin{abstract}
R-phase in a polycrystalline 50Ti48Ni2Fe shape memory alloy has been here investigated by X-Ray Powder counter Diffractometry (XRPD). With the aim to determine the $\mathrm{R}$-phase lattice parameters accurately, an original XRPD calibration method, specifically apt for shape memory alloys, has been developed. The intrinsic aberrations that affect the XRPD have been taken into account by evaluating the XRPD spectrum of the high temperature b.c.c. phase. The correction function $\Delta(2 \theta)$ has been calculated and applied to the R-phase diffraction peaks. A full indexing of the X-ray spectrum of the R-phase, not achievable by raw experimental data, has thus been obtained and the lattice parameters calculated on a set of reflections in un-aberrated angular positions with an accuracy of $\approx 1$ part in 10000 . The calibration method here developed can be used also in the XRPD study of crystalline materials that undergo a first solid-solid phase transition.
\end{abstract}

\section{INTRODUCTION}

The shape memory alloys (SMA) rely their unusual mechanical properties (shape memory and pseudoelasticity) on the thermoelastic martensitic transformation (TMT), a first-order solidsolid, diffusionless, athermal transformation. In NiTi alloys, critically depending upon composition and/or thermo-mechanical treatment, a rhombohedral phase (R-phase) can appear stable between the high temperature b.c.c phase (B2-phase) and the low temperature monoclinic phase (M-phase). Since the first detection of electric transport anomalies in the premartensitic region [1], the $\mathrm{R}$-phase in $\mathrm{NiTi}$ alloys has been widely investigated. Initially considered a "pre-martensitic" effect and later assessed as an independent phase from the martensitic one, the $R$-phase does exhibit precursory effects during the $B 2 \rightarrow R$ phase transformation: the softening of some elastic constants [2], the appearance of diffuse "extraspots" not in the exact $1 / 3$ positions in the electronic diffraction patterns [3,4], and the very large minimum in the electrical resistance, have been extensively studied. The break-through was given by [5] investigating a $50 \mathrm{Ti} 47 \mathrm{Ni} 3 \mathrm{Fe}$ where the $\mathrm{B} 2 \rightarrow \mathrm{R}$ transformation is well separated from the $\mathrm{R} \rightarrow \mathrm{M}$ one, on the temperature scale. Theoretical models to explain the origin of the $R$-phase transformation and the related precursory effects, have been proposed [68]. The thermoelastic nature of the $B 2 \leftrightarrow R$ transformation and the process of $R$-phase variants self-accomodation have been assessed [9-10]. Moreover, the shape memory effect and the pseudoelasticity related to the $B 2 \leftrightarrow R$ has been investigated [9,11-12] as well. Concerning tothe R-phase crystal structure itself, many investigations were also accomplished [10,13-14], 
mainly by TEM analysis. The R-phase lattice parameters have been determined [13,15-16], but a good agreement does not exist. The determination of the spatial symmetry group is still lacking, due to the absence of an accurate determination of the lattice parameters. The P31 and the P31m space groups have been proposed [14]. Both the lattice parameters and the spatial symmetry group are till now not assessed as a consequence of the fact that good $R$-phase single crystals are unavailable. The few X-ray powder diffraction (XRPD) studies had to deal with the drawbacks associated to this methodology: as is well known, in the XRPD, the diffraction profiles are modified by intrinsic geometrical and physical aberrations, that do not allow an accurate determination of the lattice parameters. Moreover, the "internal standard method" traditionally used to correct the effects of the aberrations cannot be applied in the case of the shape memory alloys, because the powder preparation deeply modifies the martensitic peaks.

In order to evaluate the intrinsic aberrations which affect the XRPD peaks of the R-phase, a new calibration method has been used here: the high temperature b.c.c. phase of the specimen under investigation has been exploited to evaluate the correction function $\Delta(2 \theta)$, which is later on applied to the $\mathrm{R}$-phase peaks. The corrected spectrum has been fully indexed and the lattice parameters have been determined, by means of a set of peaks at un-aberrated angular positions, with an accuracy of $\approx 1$ part in 10000 .

\section{EXPERIMENTAL}

A polycrystalline $50 \mathrm{Ti} 48 \mathrm{Ni} 2 \mathrm{Fe}$ (at. \%) alloy has been investigated. The specimens, cut from a cold rolled slab (thickness $=0.5 \mathrm{~mm}$ ), have been submitted to a solution treatment at $1000^{\circ} \mathrm{C}$ under vacuum for $3.6 \mathrm{ks}$, followed by water quenching. The specimen surface has been polished by emery paper (mesh 600) followed by electropolishing. The $\mathrm{B} 2 \rightarrow \mathrm{R}$ onset transformation temperature, $\mathrm{T}_{\mathrm{r}} \approx 14^{\circ} \mathrm{C}$, has been determined by a differential scanning calorimeter (DSC-7 Perkin Elmer) provided with a cooling system (Intracooler II). The DSC temperature calibration has been performed following [17]. A conventional powder diffractometer (Siemens D500), equipped with curved graphite monochromator in front of the scintillation counter (NaI(Tl), with $\mathrm{Be}$ window), and a $\mathrm{Cu}$ anode with a $2 \mathrm{~kW}$ generator has been used. A 20s counting time and a $0.02^{\circ} 2 \theta$-scan step were used. Measurements at constant temperature have been performed by a TTK Anton Paar sample holder. The specimen temperature has been measured by a Pt100 resistance temperature detector.

The raw XRPD data have been fitted by a PC program based on the simplex algorithm [18], to perform both $\mathrm{K}_{\alpha 1}-\mathrm{K}_{\alpha 2}$ and multiplets deconvolution. In the calculations, $\mathrm{CuK}_{\alpha 1}=1.5405981 \AA$ and $\Delta \lambda=0.00383 \AA$ were used. Peaks indexing has been accomplished by the PC program TREOR-4 [19] which also calculates the lattice parameters.

A figure of merit for the goodness of the indexing, similar to the one proposed by Smith and Snyder [20], was defined as:

$$
F\left(N_{\text {ols }}\right)=\frac{1}{\langle|\Delta 2 \theta|\rangle} \times \frac{N_{\text {int }}}{N_{\text {obs }}}
$$

where $\mathrm{N}_{\text {ous }}$ and $\mathrm{N}_{\text {ind }}$ are respectively, the numbers of the observed and indexed reflections and $\langle|\Delta 2 \theta|\rangle$ is the average of the absolute values of $\Delta 2 \theta, \Delta 2 \theta$ being the difference between the observed and calculated peak position.

The $R$-phase rhombohedral lattice has been indexed in the equivalent hexagonal system.

\section{RESULTS}

Fig. 1 shows the XRPD spectrum of the $\mathrm{B} 2$-phase obtained at $\mathrm{T}=+50^{\circ} \mathrm{C}$. In the inset, the angular position and the (hkl) indexes of each peak are reported. Fig.2 shows the R-phase spectrum at $\mathrm{T}=-32^{\circ} \mathrm{C}$, reached on cooling from the $\mathrm{B} 2$ phase. Table I reports the angular positions $2 \Theta-$ OBS of the R-phase peaks deconvoluted with the simplex algorithm. The indexing obtained on 


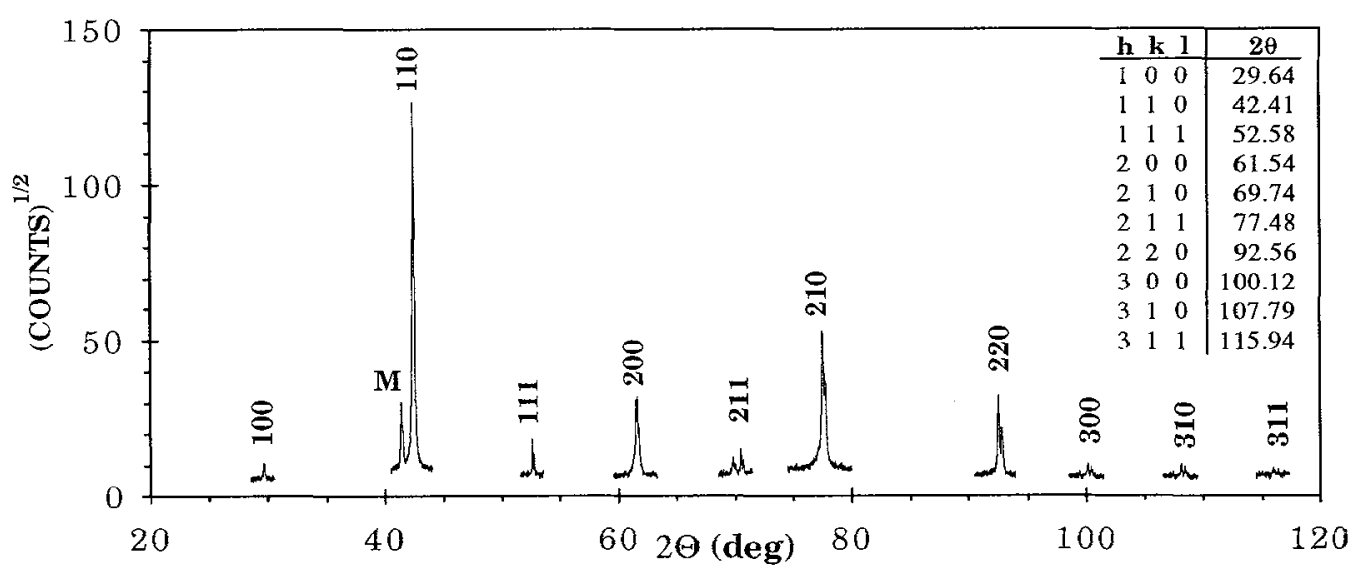

Fig.1: XRPD pattern for the B2-phase of the 50Ti48Ni2Fe specimen

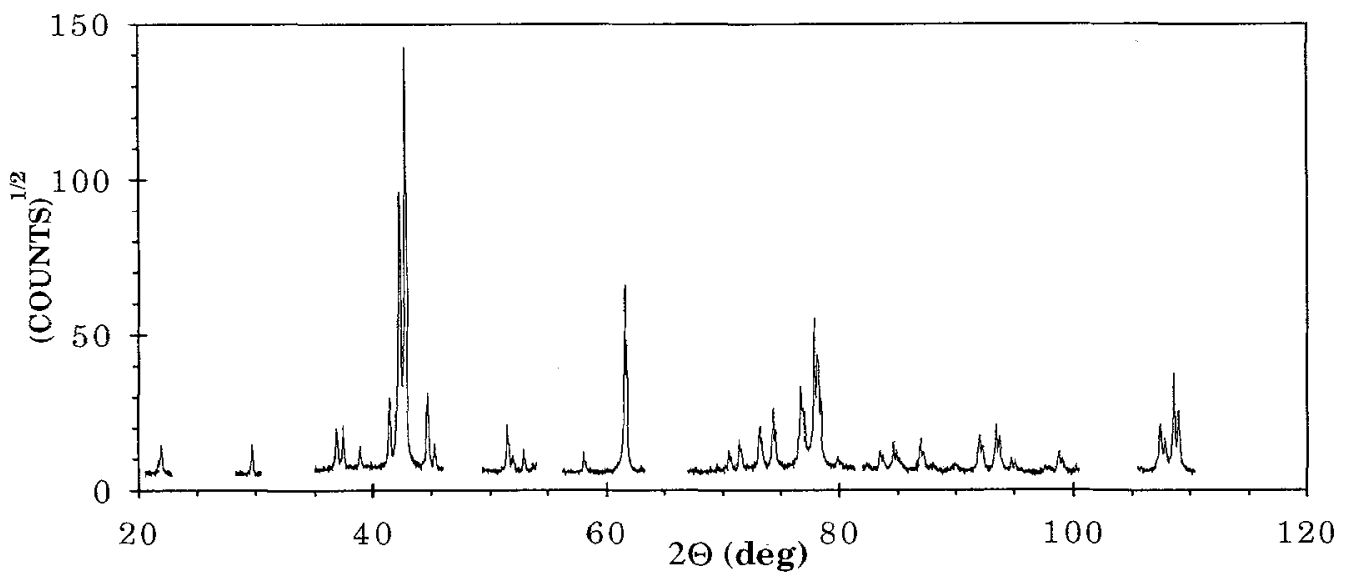

Fig.2: XRPD pattern for the R-phase of the 50Ti48Ni2Fe specimen

the $2 \Theta$-OBS peak positions, and the corresponding calculated lines are also reported in Table I. The asterisks indicate reflections not indexed by the program: it appears that only 20 reflections over 28 observed ones are indexed, with F28=62. The incomplete indexing is not surprising because the aberrations affect the angular positions of the Bragg peaks experimentally observed. In order to obtain the full indexing, the peak positions have been corrected by a proper correction function $\Delta(2 \theta)$, calculated by the following calibration method.

\section{THE CALIBRATION METHOD}

In the XRPD, the intrinsic shape of a diffraction peak, is modified as a consequence of both geometrical aberrations, related to the system specimen-diffractometer, and to physical aberrations, related to the specimen. A mathematical modelling of the XRPD aberrations has been worked out by Wilson and Parrish [21-22]: the "surface specimen displacement", the "flatspecimen" and the "specimen transparency" errors have been proved the most relevant ones. The first two are related to the non-ideal focal position of the specimen surface, while the last is connected with the specimen absorption.

Under standard measuring conditions, the above errors have been quantified by the authors [23]: the "specimen transparency" and the "flat specimen" errors are negligible; the 
"specimen surface displacement" error is the main source of aberration, inducing an error $\Delta(2 \theta)$ in the peak position given by:

$$
\Delta(2 \theta)=-2 s R^{-1} \cos \theta=-2 k \cos \theta
$$

where $\mathrm{s}$ is the misalignment of the specimen surface in a direction normal to the surface itself, in the plane of the diffraction circle, of radius $R$ (the circle centered on the specimen where both the source and the detector move). It must be pointed out that the constant $\mathrm{k}$ is not determined a priori, as s is unknown.

Following [23], $\mathrm{k}$ can be calculated by using the high temperature B2 structure. In fact, it is well known that in cubic systems the error $\Delta(2 \theta)$ in the determination of the Bragg peaks positions is related to the error in the determination of the lattice parameter $a$, through the relation:

From eq.(1) and (2),

$$
\frac{\Delta \mathrm{a}}{\mathrm{a}}=-\Delta \theta \cot \theta
$$

$$
a=a_{o}(1+k \cos \theta \cot \theta)
$$

Both $a_{o}$ and $\mathrm{k}$ can be determined by linear regression of the a(hkl), calculated by the Bragg law, as a function of $\cos \theta \cot \theta$, where $\theta$ is the corresponding $\theta(\mathrm{hkl})$.

This is shown in fig.3, where the a(hkl) calculated from the B2 reflections are reported as a function of the $\theta(\mathrm{hkl})$ and function (3) is drawn as a result of the linear regression $(\mathrm{k}=-0.00046$, $a_{o}=3.0149 \AA$ ).

A basic hypothesis is to be pointed out: the specimen-diffractometer set must be the same during the acquisition of both the B2 and R-phase spectra. That means the specimen must not be moved from the sample holder between the measurements, otherwise a different $\mathrm{s}$ could be involved. Once $\mathrm{k}$ is calculated, the angular position of the R-phase diffraction peaks can be modified using the correction function (1) as:

$$
2 \theta_{\text {carr. }}=2 \theta_{\text {exp }}-\Delta(2 \theta)=2 \theta_{\text {exp }}+2 \mathrm{k} \cos \theta_{\text {exp }}
$$

where $2 \theta_{\text {exp }}$ and $2 \theta_{\text {corr }}$, are the angular positions of the observed and corrected peaks, respectively.

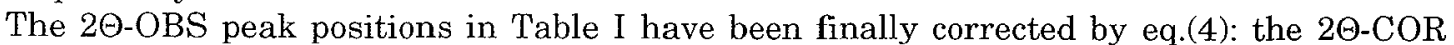
angular positions are reported in Table II with the indexing obtained on the new angular positions. The comparison between Table I and II evidences that on the corrected data:

- the number of indexed reflections increases;

- the figure of merit of the indexing increases;

- the standard deviations of the lattice parameters $a$ and $c$ decrease.

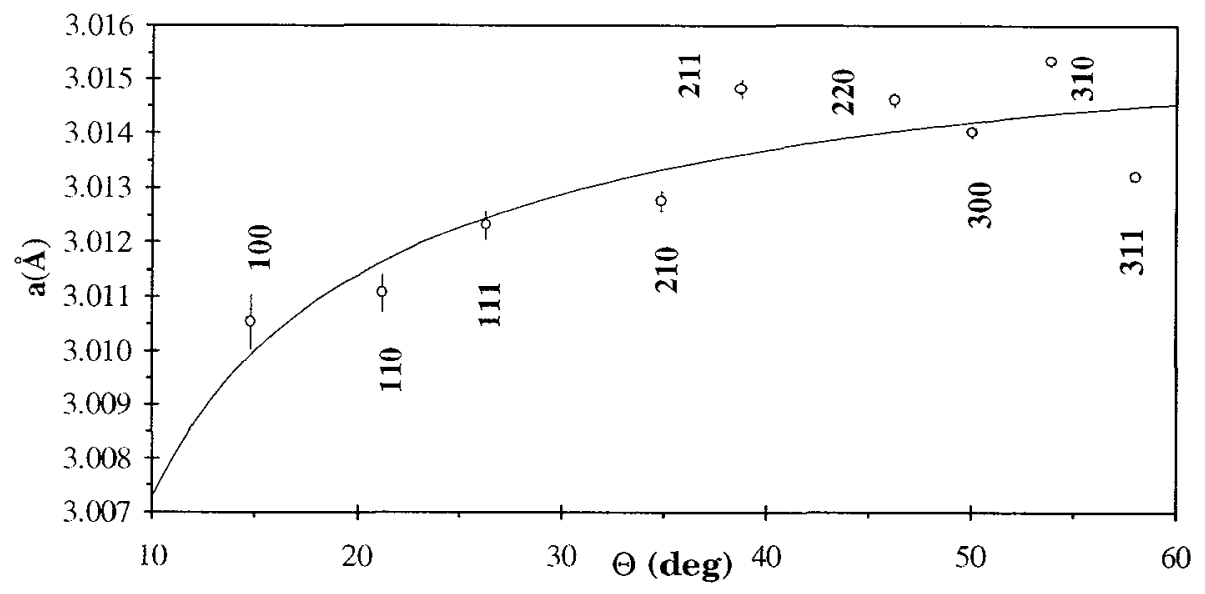

Fig.3: Interpolation curve for the b.c.c. B2-phase lattice parameter a as calculated from experimental $2 \Theta$ Bragg peaks 
Using the hexagonal basis, the lattice parameters have been determined as $\alpha=7.3321 \AA \pm 0.0003 \AA$, $c=5.2804 \pm 0.0003 \AA$. The present results are compared with the literature ones as it can be seen in Table III: in the literature data, the accuracy is lower than in the present results and the standard deviations are never given. The differences between data could be attributed to the different atomic composition of the alloys. Another source of discrepancy could be related to the temperature dependence of the rhombohedral angle (and, as a consequence, of both $a$ and $c$ ), which depends upon $\left(\mathrm{T}-\mathrm{T}_{\mathrm{r}}\right.$ ) [24], where $\mathrm{T}$ is the test temperature. This information should always be given with the structural determination of the R-phase lattice parameters. In the present case, $\left(\mathrm{T}-\mathrm{T}_{\mathrm{r}}\right.$ ) was $46^{\circ} \mathrm{C}$.

As a concluding remark, the XRPD calibration method here presented can be used for systems undergoing a first order allotropic phase transition between ordered structures: if the Miller indexes of at least one phase are known, the calibration function $\Delta(2 \theta)$ can be calculated.

Table I

Table II

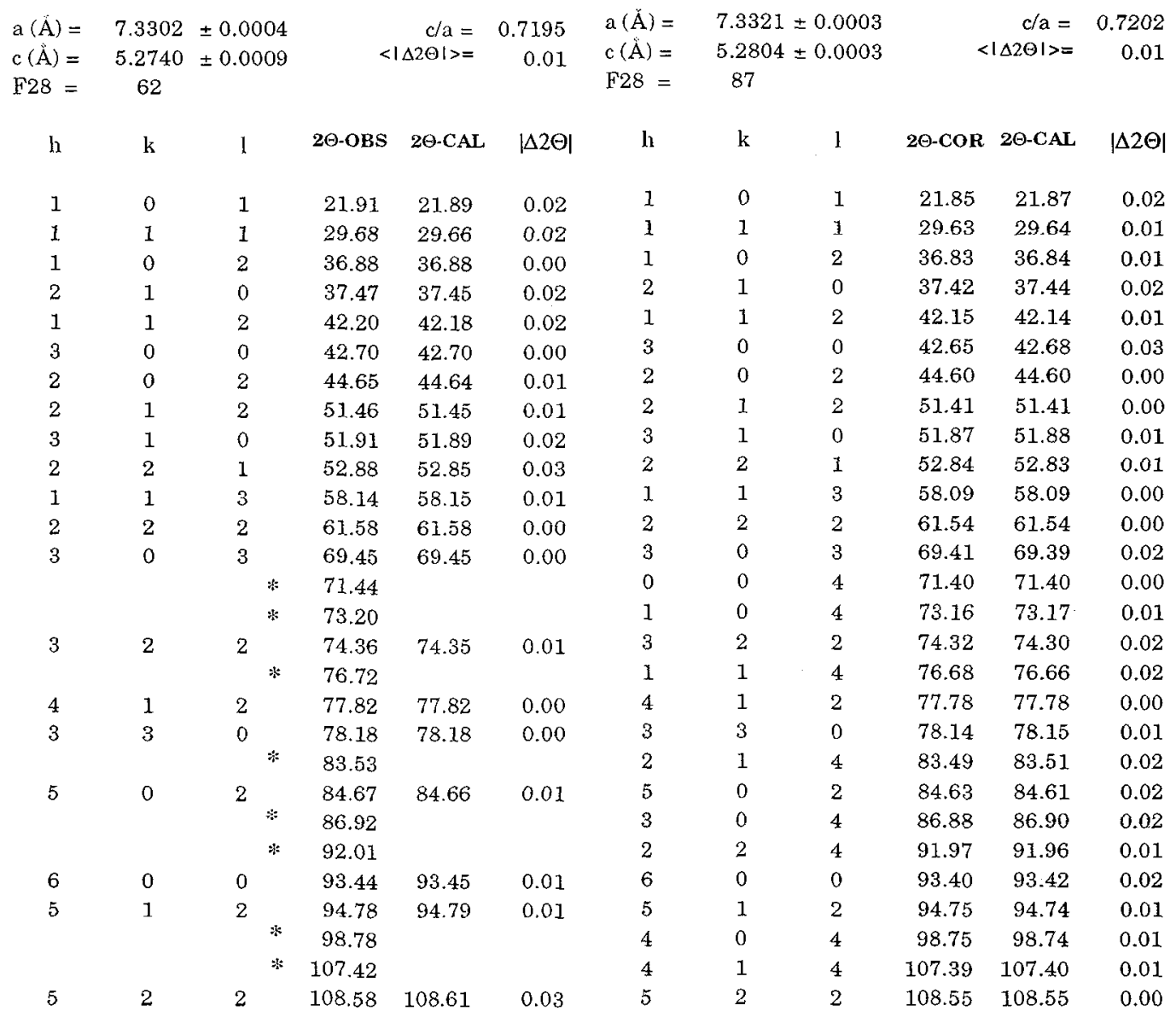

Table III

$\begin{array}{cccccccc}a & \sigma & c & \sigma & c / a & \text { ALLOY } & \text { METHOD } & \text { Ref. } \\ (\AA) & (\AA) & (\AA) & (\AA) & (\AA / \AA) & & & \\ 7.38 & = & & = & 0.7209 & 50 \mathrm{Ti} 47 \mathrm{Ni} 3 \mathrm{Fe} & \text { TEM } & {[13]} \\ 7.355 & = & & = & 0.7183 & 50.3 \mathrm{Ti} 48.2 \mathrm{Ni} 1.5 \mathrm{Fe} & \text { XRD } & {[15]} \\ 7.32 & = & & = & 0.7199 & 50 \mathrm{Ti} 50 \mathrm{Ni} & \text { XRD } & {[16]} \\ 7.3321 & 0.0003 & 5.2804 & 0.0003 & 0.7219 & 50 \mathrm{Ti} 48 \mathrm{Ni} 2 \mathrm{Fe} & \text { XRD } & \text { Present results }\end{array}$




\section{CONCLUSION}

Thanks to the original XRPD calibration method here developed, the pattern of the R-phase of a $50 \mathrm{Ti} 48 \mathrm{Ni} 2 \mathrm{Fe}$ specimen has been fully indexed. Using the hexagonal basis, the lattice parameters have been determined as $\alpha=7.3321 \AA \pm 0.0003 \AA, c=5.2804 \pm 0.0003 \AA$ at $T-T_{r}=46^{\circ} \mathrm{C}$. The method here developed can in principle be applied to any crystalline material undergoing a first order solid-solid phase transformation.

\section{ACKNOWLEDGEMENTS}

The research has been supported by P.F. MSTA (Progetto Finalizzato Materiali Speciali per Tecnologie Avanzate del CNR) of the National Research Council.

Thanks are due to Prof. K. Otsuka for providing the NiTiFe alloy.

\section{REFERENCES}

[1] Dautovich D.P. and Purdy G.R., Can.Metall.Quart. 4 (1965) 129-143.

[2] Hasiguti R.R and Iwusaki K., J.Appl.Phys. 39 (1968) 2182.

[3] Chandra K. and Purdy G.R., J.Appl.Phys. 39 (1968) 2176-2181.

[4] Sandrock G.D., Perkins A.J. and Heheman R.F., Metall.Trans. 2 (1971) 2769-2781.

[5] Matsumoto M. and Honma T., "Martensitic Transformation Intermetallic Compound $\mathrm{Ti}_{50} \mathrm{Ni}_{47} \mathrm{Fe}_{3}{ }^{\prime \prime}$, New Aspects of Martensitic Transformation, Kobe 10-12 may 1976 (The Japan Institute of Metals, 1972) pp.199-204.

[6] Hwang C.M.,Meichle M., Salamon M.B. and Wayman C.M. Phil.Mag.A 47(1) (1983) 9-30.

[7] YamadaY., Metall. Trans. A 19 (1988) 777-781.

[8] Michael G.M., Moine P. and Sinclair R., ActaMetall. 30 (1982) 125-138.

[9] Miyazaki S. and Wayman C.M., ActaMetall. 36(1) (1988) 181-192.

[10] Fukuda T., Saburi T., Doi K. and Nenno S., Mat. Trans JIM 33(3) (1992) 271-277.

[11] Stachowiak G.B. and McCormick P.G., ActaMetall. 36(2) (1988) 291-297.

[12] Miyazaki S., Kimura S. and Otsuka K., Phil.Magaz.A 57(3) (1988) 467-478.

[13] Goo E.R. and Sinclair R., ActaMetall. 33 (1985) 1717-1723.

[14] Hara T., Ohba T., Nenno S and Otsuka K., IUMRS-ICAM Tokio (1993)

[15] Saburi T., Doi K. and Nenno S., "Self-accomodation of R-phase in Ti-Ni-Fe and Ti-Ni-Al Alloys", Martensitic Transformations, Sydney 3-7 July 1989 (Trans Tech Publications, 1990) pp. 161-616.

[16] Agafanov V., Legendre B., Dubertret A., Dubois G. and Rizzo B., "In situ X-Ray Diffraction Observations of Phase Transitions in Ti49Ni51, Ti49Ni48Fe3 and Ti47Ni51Zr2 Alloys as a Function of Temperature and Tensile Stress", Martensitic Transformations, Sydney 37 July (Trans Tech Punblications, 1990) pp. 625-630.

[17] Airoldi G., Riva G., Rivolta B. and Vanelli M., J.Thermal.Anal. 41 (1994) 1-11.

[18] Mohamed J.L. and Walsh J.E., Numerical Algorithms (Clarendon Press, Oxford, 1986) pp. 220-274

[19] Werner P.E., Z.Krist. 120 (1964) 374.

[20] Smith G.S. and Snyder R.L., J.Appl.Cryst. 12 (1979) 60.

[21] Wilson A.J.C., Mathematical Theory of X-ray Powder Diffractometry (N.V. Philips' Gloeilompenfabrieken-Eindhoven-Netherland, 1963)

[22] Parrish W. and Wilson A.J.C., X-ray analysis papers (Centrex Publishing

Company- Eindhoven-Netherland, 1965) pp. 142-

[23] Airoldi G., Riva G. and Vanelli M., to be published.

[24] Meichle M., Ph.D.Thesis, University of Illinois, Urbana Champaign (1981) 